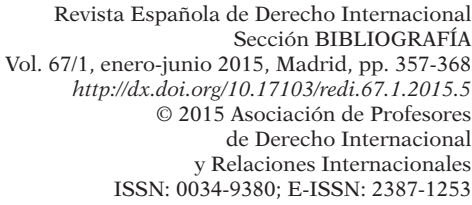

\title{
V. BIBLIOGRAFÍA
}

Sección coordinada por C. EsPósito (Derecho internacional público), E. ZaBALO (Derecho internacional privado) e I. RodRíguez MANZANo (Relaciones Internacionales)

\section{A) RECENSIONES}

BARBÉ, E. (dir.), La Unión Europea en las relaciones internacionales, Madrid, Tecnos, 2014, 433 pp.

La Prof. de la Universidad Autónoma de Barcelona y coordinadora del programa de investigación en el Institut Barcelona d'Estudis Internacionals (IBEI), Esther Barbé, es la directora de esta nueva contribución al estudio de la acción exterior de la Unión Europea (UE) en sus múltiples manifestaciones. Según nos explica en su primer capítulo que ha escrito como marco introductorio, esta obra colectiva aborda el estudio de la acción exterior de la UE desde el ámbito de las Relaciones Internacionales y del Análisis de la Política Exterior, pero también se cuenta con el análisis de esta realidad desde las perspectivas que ofrecen el Derecho Europeo y la Economía Política Internacional, lo que en última instancia impregna a esta obra de cierto carácter interdisciplinar. De hecho, si se repasa el elenco de autores que participan en su redacción, organizados en torno al Observatorio de Política Exterior Europea (Grupo de investigación consolidado reconocido por la Generalitat de Catalunya, 2009, SGR-990), se puede comprobar que proceden de diversas disciplinas científicas y/o ámbitos profesionales (Re- laciones Internacionales, Derecho internacional público, Economía...).

Esta obra colectiva, además del citado capítulo I, de carácter introductorio, se estructura en Tres Partes, con un total de XIX capítulos, que en conjunto ofrecen una visión actual y crítica de las principales manifestaciones a través de las que se desarrolla el obrar internacional de la UE, como subsistema internacional de ámbito regional europeo. La Primera, dedicada al estudio de las "Instituciones, procesos e instrumentos», está formada por los capítulos redactados por Alfonso González Bondía (II: «La acción exterior en el Tratado de Lisboa»), Pol Morillas (III: «Actores y procesos en la Política Exterior y de Seguridad Común»), Débora Miralles Solé (IV: «Instrumentos de acción exterior»), Milagros Álvarez Verdugo (V: «Actores, procedimientos y capacidades de la Política Común de Seguridad y Defensa»), Raúl Hernández i Sagrera (VI: "Instrumentos de acción exterior en Justicia, Libertad y Seguridad»), y Laia Mestres (VII: «La europeización de las políticas exteriores nacionales»). 
La Segunda Parte, titulada "Cartografía de las relaciones exteriores», está compuesta por los capítulos responsabilidad de Elisabeth Johansson-Nogués (VIII: "Ampliación de la Unión Europea»), Michal Natorski y Eduard Soler i Lecha (IX: «Relaciones de la Unión Europea con los vecinos»), Federico Guerrero (X: «Relaciones de la Unión Europea con el mundo en desarrollo»), Lluc López i Vidal, Francesc Serra y Juan Pablo Soriano (XI: «Relaciones de la Unión Europea con los socios estratégicos: Estados Unidos, Rusia y China»), y Lluc López i Vidal y Juan Pablo Soriano (XII: «La Unión Europea y el interregionalismo»).

Por último, la Tercera Parte se ocupa del papel de la UE como «Actor en la gobernanza global», y se estructura en un total de siete capítulos escritos por $\mathrm{Pa}$ tricia García-Durán y Montserrat Millet (XIII: «La Unión Europea en el comercio internacional —OMC—»), José Antonio Sanahuja (XIV: «La Unión Europea en las finanzas globales — G20 y FMI—»), Oriol Costa (XV: «La Unión Europea en las negociaciones internacionales del clima»), Anna Herranz Surallés (XVI: «La Unión Europea en la fragmentaria gobernanza global de la energía»), Benjamín Kienzle (XVII: «La Unión Europea y la política de no proliferación de armas de destrucción masiva»), Esther Barbé (XVIII: «La Unión Europea como promotora de derechos humanos en Naciones Unidas») y Martijn C. Vlaskamp (XIX: «La Unión Europea frente a las autoridades privadas»).

Con carácter general, esta obra nos ofrece un conjunto de contribuciones dedicadas al estudio de la acción exterior europea que aúnan tanto la información actualizada y bien documentada, como el análisis crítico, de los distintos temas de los que se ocupan. Con sus estudios los autores ponen de manifiesto tanto las dificultades como los logros que presenta el desarrollo de la acción exterior de la Unión Europea, como organización internacional de integración europea, que como es sabido coexiste y se interrelacio- na estrechamente con las políticas exteriores de sus 28 Estados miembros.

Como ya se ha señalado, se puede predicar el carácter en cierta medida interdisciplinar de esta obra, teniendo en cuenta la diversa procedencia académica y/o profesional de sus autores. Si bien es cierto que son mayoría los autores de esta obra que centran su actividad académica y/o profesional preponderantemente en el ámbito de la disciplina de las Relaciones Internacionales. Lo que quizás sirva de explicación al hecho de que apenas se pueden contabilizar citas de la jurisprudencia del Tribunal de Justicia -y de la doctrina que se ha dedicado a su análisis- a lo largo de sus $433 \mathrm{pp}$. A pesar de que la labor del principal órgano jurisdiccional de la UE ha tenido y sigue teniendo su relevancia en el ámbito de la acción exterior de esta organización internacional europea. En particular, la omisión casi completa de referencias a la jurisprudencia del Tribunal de Justicia sobre todo se hace muy patente en los capítulos de la Primera Parte dedicados a los aspectos jurídicos e institucionales de la acción exterior de la UE, en concreto en los citados capítulos II, III, IV y VI.

Por lo que se refiere a su factura formal, la obra presenta una edición muy cuidada, como suele ser habitual en las publicaciones de la Editorial Tecnos, una Editorial de prestigio muy consolidada en el ámbito de las ciencias sociales y jurídicas. Si bien, se echa en falta que en un libro colectivo de estas características (XIX capítulos, con un total de 433 pp.), no se incluya tanto un listado de las abreviaturas más utilizadas, como los índices finales que suelen ser más comunes (Bibliografía, acuerdos internacionales...), por lo menos el de materias, que podrían ser de cierta utilidad para cualquier lector.

Cabe destacar a este último respecto, que en todos los capítulos se incluye un último apartado titulado «Bibliografía de referencia», en el que se recogen las cinco contribuciones que el autor considera de 
mayor relevancia sobre el tema que versa el capítulo en cuestión. Aunque también se debe señalar que en algunas de las «bibliografías de referencia» que se proponen (capítulos escritos por Pol Morillas, Débora Miralles Solé, Raül Hernández i Sagrera, Laia Mestres, Elisabeth Johansson-Nogués, Michal Natorski y Eduard Soler i Lecha, Federico Guerrero, Benjamin Kienzle y Martijn C. Vlaskamp) se opta por la cita en exclusiva de doctrina publicada en inglés, en detrimento de la doctrina escrita no sólo en español sino también en otras lenguas románicas de amplia difusión (francés...). Si ya suele ser muy excepcional que la doctrina internacionalista escrita en inglés incluya referencias bibliográficas en otras lenguas, incluido el español, en opinión del autor de esta recensión resulta del todo contraproducente que la doctrina escrita en español demuestre también una clara tendencia a relegar al olvido a la doctrina que no publica en inglés a la hora de proponer una «bibliografía de referencia».
No obstante esta última consideración, que sin duda puede ser objeto de debate académico, en conjunto como ya se ha dicho con esta obra se ofrece información y análisis actualizados sobre buena parte de las múltiples facetas que presenta la acción exterior de la UE, desde una perspectiva en cierta medida interdisciplinar. No se puede negar, por tanto, el encomiable esfuerzo llevado por sus autores con la publicación de este libro colectivo, bajo la dirección de la Prof. ${ }^{a}$ Esther Barbé. En definitiva, esta obra supone una nueva aportación de la doctrina publicada en lengua española al debate sobre los avances y limitaciones que presenta a fecha de hoy la actuación de la UE en su obrar internacional, y constituye un libro de referencia para todos aquellos que estén interesados en esta temática.

Jaume FERRER LLORET

Universidad de Alicante

http://dx.doi.org/10.17103/redi.67.1.2015.5.01

Estrada Adán, G. E. y Fernández de Casadevante Romaní, C. (coords.), Manual de Derecho internacional de los derechos humanos, 1. a ed., México, Porrúa, 2014, 455 pp. y FERnández dE CASAdEVAnTe Romaní, C. (dir.), Nuevos desarrollos en el Derecho internacional de los derechos humanos: los derechos de las víctimas, Pamplona, Thomson Reuters Aranzadi, 2014, 287 pp.

1. El Catedrático de Derecho Internacional Público de la Universidad Rey Juan Carlos de Madrid (URJM), Carlos Fernández de Casadevante, muy recientemente ha co-coordinado en un caso y dirigido en otro, la edición de dos volúmenes colectivos en materia de Derecho internacional de los derechos humanos. Para quienes siguen las obras de juristas internacionalistas españoles en ese ámbito, en verdad destacables por su número y calidad dentro del panorama de la doctrina jusinternacionalista mundial, el perfil del autor y su obra son bien conocidos. Personalmente me congratulo de las aportaciones de este autor que sobrepasan ya ampliamente la intención pedagó- gica (personalmente doy a esa dimensión una gran importancia), para entrar en el campo de la especialización y análisis consiguiente de los progresos en temas o ámbitos específicos determinados.

2. El «Manual» de «Derecho internacional de los derechos humanos» es el fruto de una colaboración académica y editorial entre profesores de la UNAM y de la Universidad Rey Juan Carlos de Madrid $\mathrm{y}$, como sus coordinadores señalan, tiene el propósito de ofrecer a los estudiantes iberoamericanos una herramienta que contenga la información sustancial del Derecho internacional de los derechos humanos. En 18 lecciones se presentan 
así, de modo sucinto, completo y cabal, el marco general de la protección jurídica internacional de los derechos humanos y su protección organizada a través de diferentes subsistemas de base convencional, junto a temas relativos a la protección de los derechos de personas y grupos vulnerables y a la protección frente a determinadas violaciones o crímenes internacionales específicos. Siete de los temas han sido escritos por los dos coordinadores y el resto (a veces en doble autoría) por hasta doce profesores, provenientes fundamentalmente de la UNAM, la URJM y la Universidad Complutense de Madrid.

3. Como todo Manual éste es y será siempre susceptible de perfeccionamientos a medida que su eficacia vaya siendo probada en la práctica docente y en su aportación a la adecuada formación de los estudiantes. Así, pienso que la protección de los derechos de la mujer merece un capítulo especial, separado de la protección de los derechos humanos de las personas LGTB. Por otro lado, como presidente que he sido del Comité contra la tortura de Naciones Unidas y miembro suyo durante doce años puedo recomendar con fundamento la inclusión de mayores referencias a la jurisprudencia del Comité, relevante en temas cruciales como, por ejemplo, el de la licitud de las medidas adoptadas por los Estados contra todas las formas de terrorismo.

Muchos años antes de que el Comité de Inteligencia del Senado de Estados Unidos informara públicamente de la comisión de torturas en lugares de privación de libertad bajo el control de la CIA (2014), el Comité contra la tortura ya había exigido inter alia en sus Recomendaciones Finales de 2005 a Estados Unidos (tras analizar su segundo Informe) el cierre inmediato de la prisión de Guantánamo. El Manual podrá seguramente en ediciones posteriores enriquecerse con la cita de casos y supuestos de la práctica, muy cuidadosamente seleccionada debido a su prolijidad y relevancia, como por lo demás ya se hace en diversos de los trabajos que integran la publicación.

4. Por lo que refiere al segundo de los volúmenes aquí reseñados cabe decir que la protección jurídica internacional de los derechos de las víctimas de delitos y en especial de violaciones de normas internacionales e internas protectoras de los derechos humanos se ha constituido en verdad en un ámbito específico que cabe calificar, como pertinentemente destaca el título del libro, de «nuevo desarrollo» del Derecho internacional de los derechos humanos. Como destaca el Dr. Fernández de Casadevante en el prólogo, el concepto de "víctima» aquí relevante es transversal a todos los sectores del Derecho internacional que protegen derechos humanos y ya sean las conductas lesivas atribuibles a uno o más Estados, a actores no estatales o incluso a individuos; es además relevante con relación a las víctimas de delitos punibles según los ordenamientos estatales.

De ahí que, sobre la base de la práctica, quepa identificar regímenes relativos a categorías concretas de víctimas. De ahí también que estemos ante un campo normativo de muy importante repercusión lógicamente en los Derechos internos, que deben tener en cuenta y aplicar los principios y normas que en este ámbito les vinculan. Los autores hacen un esfuerzo para elaborar una teoría de los «derechos de las víctimas» que los Estados deben proteger articulando ese campo jurídico sobre la base de principios que por lo que se refiere al ámbito universal arrancan de la Resolución 40/34 de la Asamblea General de 29 de noviembre de 1985 en la que se contiene la «Declaración sobre los principios fundamentales de justicia para las víctimas de delitos y abusos de poder».

5. Este ámbito jurídico, como se destaca en diferentes contribuciones al volumen hechas por profesores de varias Universidades españolas (Rubén Carnerero, Anna Gemma López, Virginia Mayordo- 
mo, Julia Ruiloba Alvariño y Marina Sanz-Díez de Ulzurrun) había quedado cubierto tradicionalmente (por lo que se refiere al Derecho internacional) por el régimen relativo a las conductas que un Estado declarado internacionalmente responsable de la violación de derechos humanos, debía realizar para reparar los daños causados a las víctimas.

6. La obligación internacional de reparar los daños causados por violaciones de derechos humanos ha sido en verdad dotada crecientemente por los órganos internacionales controladores o enjuiciadores del respeto a las normas del Derecho internacional, de contenidos más y más precisos (véase, por ejemplo, la Observación general núm. 3 del Comité contra la tortura de Naciones Unidas adoptada en 2012).

7. Por lo demás, quedan aquí por explorar y determinar más en profundidad, en la medida en que la práctica lo va permitiendo, los principios y reglas y en general los regímenes aplicables a las demandas de responsabilidad civil debida a las víctimas de un hecho internacionalmente ilícito o, en su caso, un delito; particularmente las víctimas de los delitos de relevancia internacional, tipificados por medio del Derecho internacional. No lejos se encuentra entonces una referencia al régimen del ejercicio extraterritorial de la jurisdicción interna, civil que permita la exigencia «universal» de reparación incluso a Estados extranjeros, en principio inmunes, por las violaciones más graves del Derecho internacional (genocidio, crímenes de guerra y crímenes contra la Humanidad).

Las referencias al Derecho español en la materia y el capítulo dedicado específicamente a la protección de las víctimas del terrorismo redondean esta destacable y bienvenida publicación.

Fernando M. MARIÑo

Universidad Carlos III de Madrid

http://dx.doi.org/10.17103/redi.67.1.2015.5.02

\section{Gandía SelLENS, M. A., El arbitraje frente a los litigios internacionales en ma- teria de propiedad intelectual: la arbitrabilidad y la adopción de medidas cautelares, Cizur Menor, Aranzadi, 2014, 231 pp.}

1. La monografía que aquí se analiza, constituye la trigésimo segunda de la serie de monografías asociadas a la Revista Aranzadi de Derecho Patrimonial, estando prologada por el Prof. Dr. Guillermo Palao Moreno, Catedrático de Derecho internacional privado de la Universidad de Valencia. Esta obra monográfica tiene como objeto el estudio, análisis y valoración de la utilización del arbitraje como método de solución de las controversias internacionales en materia de propiedad intelectual (aunque ciertamente una vez visto su contenido, su título pudiera llevarnos un poco a equívoco, pues no sólo se refiere a los litigios relativos a los derechos de autor, sino que engloba igualmente también los atinentes a la propiedad industrial).
2. Aunque en la obra reseñada se realiza una primera y breve aproximación global a la materia citada, sin embargo, la autora se centra, en profundidad, en estudiar y analizar dos de los aspectos más específicos y de gran importancia en relación con los litigios planteados en este ámbito: por una parte, la controvertida cuestión de la arbitrabilidad de las pretensiones derivadas de los derechos de propiedad intelectual en el ámbito internacional y, por otra parte, la necesaria tutela cautelar en los procedimientos arbitrales internacionales sobre derechos de propiedad intelectual.

3. La elección de centrar el estudio en los dos citados temas no es una cuestión baladí, tal y como nos señala acerta- 
damente la autora. En efecto, en primer lugar, un problema de gran envergadura que se plantea en la relación arbitraje internacional con las controversias en materia de propiedad intelectual, es el relativo a la susceptibilidad para someter a dicho método de solución de controversias este tipo de disputas que cuentan con una serie de características específicas; a ello se añade, en segundo lugar, además, la especial configuración de la tutela cautelar en un proceso arbitral internacional, más aún teniendo en cuenta la especial relevancia e importancia que la misma cuenta en los litigios internacionales relativos a los derechos de propiedad intelectual e industrial.

4. La estructura de la obra se divide en tres capítulos, contando además con una introducción y las conclusiones. Así, de este modo el lector encuentra, en primer lugar, como resulta casi habitual, una necesaria presentación de la obra, en donde se acota el objeto de análisis, poniendo de manifiesto la importancia y la actualidad de la materia objeto de estudio, así como estableciendo la metodología que se va a seguir en el resto de la monografía.

5. Seguidamente, el capítulo primero se dedica a presentar al lector ciertas cuestiones generales de la materia objeto de estudio. Aunque en esta parte de la obra se abordan conceptos básicos para un especialista, sin embargo, la autora acota con mesura su discurso en este punto, centrándose en ayudar al lector no especializado a comprender la problemática que va a ser tratada más adelante. De este primer capítulo resulta de especial interés el apartado dedicado a las ventajas e inconvenientes que el arbitraje internacional presenta como método de resolución de controversias en materia de propiedad intelectual.

6. El capítulo segundo de la obra reviste un especial interés. Su importancia radica en el análisis profundo y pormenorizado que se realiza de una de las cuestiones objeto de estudio. En efecto, este segundo capítulo se centra en el estudio de la arbitrabilidad de las pretensiones derivadas de derechos de propiedad intelectual en los litigios internacionales. Con dicho objetivo, y tras dedicar un primer epígrafe a la definición y clases de arbitrabilidad, la obra considera la importante cuestión de la arbitrabilidad objetiva de la propiedad intelectual desde el prisma del régimen jurídico español. Tras ello y, una vez analizada la normativa española conjuntamente con una breve valoración comparada de otros Ordenamientos jurídicos nacionales, se aborda la problemática relación de la arbitrabilidad con el orden público, teniendo siempre presente las especiales características que la propiedad intelectual presenta. De este modo, en este apartado se analizan y valoran los argumentos a favor y en contra de la arbitrabilidad de esta materia, los cuales traen causa de las diferentes concepciones existentes sobre el orden público. Posteriormente, se incluye un apartado relativo a los distintos momentos procesales en los que se puede plantear la cuestión de la arbitrabilidad. En este sentido hay que señalar que, pese a que se trate de una cuestión puramente procesal, sin que se planteen particularidades ratione materiae, el análisis de esta cuestión se justifica tanto en aras de presentar un estudio lo más completo posible como por tener una decisiva incidencia sobre el siguiente aspecto clave analizado en la obra. En efecto, para terminar dicho capítulo segundo, la autora efectúa un análisis de la ley aplicable para determinar la arbitrabilidad de la controversia. Éste es un extremo clave, debido al gran impacto de fondo que provoca esta cuestión. Así, y como se señala en la propia obra, la concreción de dicha ley variará en función del momento en que se plantee la cuestión de la arbitrabilidad, de tal manera que el epígrafe anterior adquiere toda su razón de ser.

7. El tercer y último capítulo de la obra se dirige específicamente al trata- 
miento de la tutela cautelar, en relación con arbitrajes internacionales sobre pretensiones derivadas de derechos de propiedad intelectual. En este concreto sector de los métodos de resolución de controversias, la adopción de las medidas cautelares presenta una especial relevancia e importancia para la salvaguarda de los derechos de que se trata, debido, sobre todo, a la rápida evolución de los avances informáticos y tecnológicos. De tal forma que, en este capítulo, tras examinarse el concepto y las clases de las medidas cautelares útiles para la resolución del tipo de litigios que la monografía aborda, se pasa a analizar el poder de los árbitros para su adopción. Asimismo, se analiza la intervención judicial al respecto. Por último, se aborda el estudio en profundidad de dos tipos de medidas cautelares, a saber: las medidas ex parte y las medidas ante causam. Con ello, la autora quiere poner de manifiesto que, a pesar de que dicho tipo de medidas actualmente no tienen más que una escasa y todavía incipiente utilización en el arbitraje internacional, la promoción y facilitación de las mismas supondría una gran mejora cualitativa en la resolución de los litigios relativos a la propiedad intelectual.

8. Finalmente, la obra se cierra con cinco valientes conclusiones, que se manifiestan en favor tanto de la arbitrabilidad prácticamente absoluta de los derechos de propiedad intelectual como de la necesidad de otorgar a los árbitros las facultades suficientes para que sean verdaderamente independientes respecto a los jueces estatales en relación con la adopción de medidas cautelares. Se trata de conclusiones que, a primera vista, pudieren parecer arriesgadas pero que, sin embargo, vienen acompañadas de argumentos bien fundados y que invitan al lector a una pausada reflexión.
9. Junto a todo lo anteriormente expuesto y ya desde una perspectiva metodológica, este estudio monográfico no sólo contempla la vertiente teórica del arbitraje — recogiendo opiniones de la más especializada doctrina internacional-, sino que, además, se hace eco de la práctica arbitral en la materia - al incluir y valorar jurisprudencia arbitral procedente de distintos sistemas jurídicos y, lo que es más relevante, al estudiar alrededor de un centenar de laudos arbitrales inéditos en materia de propiedad intelectual-. En este sentido, es especialmente destacable cómo se ha recogido la práctica de una de las instituciones arbitrales por excelencia - como es la Corte de Arbitraje de la Cámara de Comercio Internacional de París-, lo que dota a la obra de un plus adicional que es difícil de observar en otros estudios académicos sobre estas cuestiones. La utilización y valoración de todos estos materiales nos muestra cómo el arbitraje ha llegado para quedarse de manera definitiva como un método esencial y preferente para la solución de las controversias internacionales en este ámbito.

10. En definitiva, pese a la juventud de su autora, la obra sorprende por el rigor científico con que se aborda la temática analizada. Y ello, no sólo porque la aproximación a la materia está a la altura de la calidad que se espera en trabajos académicos de carácter monográfico y especializado, sino por la forma de exposición especialmente clara y directa de las ideas que se pretenden transmitir. Se trata, por tanto, de una obra cuya lectura es muy aconsejable para todos aquellos interesados en la litigación internacional de los derechos de propiedad intelectual.

Enrique FERNÁNDEZ MASIÁ

Universidad de Castilla-La Mancha

http://dx.doi.org/10.17103/redi.67.1.2015.5.03 
Mangas Martín, A., Humanización, Democracia y Estado de derecho en el Ordenamiento internacional, Madrid, Real Academia de Ciencias Morales y Políticas, 2014, 214 pp. (disponible también en versión electrónica: http:// www.racmyp.es/docs/discursos/D88.pdf)

1. El 8 de abril de 2014, la Real Academia de Ciencias Morales y Políticas recibía solemnemente como académica de número a Araceli Mangas Martín, Catedrática de Derecho internacional público y Relaciones internacionales de la Facultad de Derecho de la Universidad Complutense y antes de la Universidad de Salamanca. Sucede la Prof. ${ }^{a}$ Mangas en la Medalla núm. 9 a otros dos maestros del Derecho internacional público, los Profs. Truyol Serra, que la portó entre 1972 y 2003, y Carrillo Salcedo, entre 2005 y enero de 2013. Humanización, Democracia y Estado de derecho en el Ordenamiento internacional es, precisamente, el discurso de recepción de la nueva académica, al que contestó el académico y Catedrático de Derecho administrativo, Santiago Muñoz Machado.

2. Marc Fumaroli, historiador y especialista en retórica, asimila los discursos académicos de recepción o ingreso y de contestación a una especie de espejo veneciano en el que, con gran ceremonial, se invita al nuevo académico a que se mire, después de que haya agradecido a sus pares la cooptación y hecho el elogio de sus predecesores. Y sentencia: en la imagen que de él (o de ella) propone uno de sus colegas, el nuevo académico se ve por última vez como un simple mortal, precisamente en el momento en que entra en la inmortalidad.

Vale acaso esta acepción del discurso de ingreso para la academia francesa, cuyos miembros, es sabido, son «inmortales» (y Fumaroli lo es); vale quizás para otras academias y para los discursos de otros académicos, pero no para Araceli Mangas, que optó en el suyo por romper el espejo y comenzar su discurso confiando, con Miguel de Unamuno, en «que no me ciegue la ceguera del honor», e inter- pretando la dignidad recibida como una distinción al colectivo de internacionalistas españoles.

3. Al tomar posesión como académico en 1972, el discurso de Antonio Truyol y Serra versó sobre «La integración europea: idea y realidad» (accesible en http://www.racmyp.es/docs/discursos/ D69.pdf) y se convirtió en una muestra más de su obra seminal en la doctrina iusinternacionalista española en la materia. Por su parte, en 2005, Juan Antonio Carrillo Salcedo dedicó su discurso de recepción —bajo el título de «Permanencia y cambios en Derecho internacional»- a analizar la evolución de la disciplina y de la realidad que intenta ordenar entre 1815 y 1914 , el proceso de reforma y modernización que experimentó entre 1919 y 1939 con la Sociedad de Naciones y la Organización Internacional del Trabajo, el cambio que supuso el orden internacional instaurado en 1945 y en la inmediata posguerra con la Carta de las $\mathrm{Na}$ ciones Unidas y, finalmente, los cambios que había experimentado desde entonces en su naturaleza y funciones. Aunque la calificación no se ajusta enteramente a la densidad de aquella exposición (accesible en http://www.racmyp.es/docs/discursos/D11.pdf), diríamos que el discurso del Prof. Carrillo Salcedo, una disección magistral y rigurosa del pasado, pertenecía a lo que un estudioso de la retórica clásica llamaría discurso judicial.

4. De entre las dos opciones que se le presentaban, y sin que en esta ocasión quepa sostener que elegir es renunciar a algo, la nueva académica prefirió seguir la senda de Carrillo Salcedo y abordar el Estado del Derecho internacional público en la actualidad. Su discurso, sin embargo, más que al género judicial, pertenece al deliberativo, pues aunque no 
rehúye el análisis del pasado ni del presente, como haría un discurso epidíctico, afronta decididamente el tiempo futuro y se pronuncia de modo comprometido, apasionado incluso, por un Derecho internacional institucionalizado en torno fundamentalmente a Naciones Unidas - y a la Unión Europea- que busque y garantice la justicia, la limitación del poderoso - la cita introductoria del discurso es Inde datae leges, ne firmior omnia posset (Para esto se hicieron las leyes, para que el poderoso no lo pueda todo), de Publio Ovidio Nason-y la defensa de unos determinados valores universales emergentes: la humanización, es decir, «la construcción de un Derecho internacional común de la Humanidad», la democracia y el Estado de Derecho, valores que son para Mangas Martín «el caldo de cultivo en el que es posible realizar los derechos humanos y, al fin, la paz» y que están "al servicio de valores meta en el Derecho internacional contemporáneo: paz, igualdad y desarrollo».

5. La defensa de estos valores es hoy posible porque hemos asistido a una transformación en la comunidad internacional; nos lo recuerda la Prof. ${ }^{a}$ Mangas cuando contempla el Derecho internacional de nuestros días, tan distinto, nos advierte, de «el mundo de ayer»: «Frente al eterno ayer anterior a la Segunda Guerra Mundial, vivimos desde entonces - y en una acusada aceleración histórica y tecnológica a partir de la caída del muro de Berlín-, una continua transformación de la comunidad internacional, de su ordenamiento y de la condiciones de su aplicación [...]. En efecto, el Derecho internacional hace décadas que no se limita a distribuir competencias (territoriales y personales) y a regular la mera coexistencia entre Estados. El Derecho internacional de nuestros días hace tiempo que ha llevado cada vez más lejos la progresiva institucionalización de sus funciones y su ampliación a nuevos ámbitos, lo que de por sí es un cambio transcendental en relación a siglos anteriores. Además, sus nuevos objetivos giran en torno a valores que van más lejos del formal mantenimiento de la paz y seguridad colectiva y se adentran en la organización interna de los Estados y en las relaciones de éstos con la ciudadanía».

Esta transformación lleva a concluir a la académica Mangas que «el Derecho internacional de nuestros días es más un faro que guía y da esperanza a la Humanidad (el derecho deseable) que la fotografía de la realidad en toda su crudeza (el derecho vivido) [...]. El nuevo Derecho internacional, tanto el soft law o derecho declarativo o programático como el hard law consuetudinario y convencional que se está creando en las últimas décadas, regula utopías pendientes de materialización en las que se avanza a duras penas».

6. Y al estudio de esta tensión entre el deber-ser y el ser, entre el horizonte utópico y la práctica que día a día construye un Derecho al servicio de los valores universales que deben presidir la articulación de la sociedad internacional, a «recorrer esas zonas de colisión entre las consecuencias de los nuevos valores y las normas que los protegen (en materia de derechos humanos, democracia y Estado de Derecho en el plano internacional) con las clásicas normas internacionales que permanecen en vigor», dedica la Prof. a Mangas el desarrollo central de su discurso.

El camino no ha sido fácil, y aunque ha propiciado «mutaciones de la Carta ocasionando la ampliación de las competencias coercitivas del Consejo de Seguridad», está lleno de "disfunciones, dificultades y, en ocasiones, hasta contradicciones y frustrantes vacíos en relación con normas clásicas del Derecho internacional», que Araceli Mangas expone con rigor y espíritu crítico, sin ahorrar al lector el juicio severo que le merece la práctica de algunos Estados o la del propio Consejo de Seguridad de Naciones Unidas, y sin dejar de mostrar impaciencia 
por el retraso que esas conductas producen en el advenimiento de los valores que comparte y defiende con ardor.

Y así, repasa la Prof. ${ }^{a}$ Mangas de modo exhaustivo supuestos recientes de graves violaciones de derechos humanos y las acciones del Consejo de Seguridad ante ellas, y se pregunta si un conflicto armado interno es motivo suficiente para la intervención armada del Consejo de Seguridad o si los derechos humanos no son en ocasiones una coartada para intervenciones ilegítimas; analiza el consentimiento del Estado a la asistencia humanitaria, los desajustes normativos en el proceso de formación de la obligación de proteger y las fortalezas y debilidades de la doctrina sobre esta responsabilidad de proteger, y estudia los desajustes estructurales que se dan en la relación entre la responsabilidad internacional por violaciones de derechos humanos y las inmunidades soberanas, ámbito en el que postula inequívocamente que «la soberanía no puede servir de pretexto para entorpecer la rendición de cuentas eficaz de los Estados por tales crímenes».

7. A continuación, defiende Araceli Mangas que la democracia, bien jurídico internacional, es un valor instrumental al servicio de la paz, su condición de posibilidad: "Donde hay democracia no hay conflicto armado interno en el seno de esa sociedad», "entre democracias no hay guerras: un Estado democrático no agrede a otro Estado democrático», y expone los factores que han propiciado su inserción en el ordenamiento internacional, entre otros la dilución del conflicto Este-Oeste, aunque también los que la amenazan: "Fenómenos sociales y políticos diversos tales como gobierno autocrático, gobierno ineficiente y sin control parlamentario efectivo, manipulación de orden constitucional y legal, corrupción, falta de sanción a las infracciones penales causadas por las autoridades o por los grupos organizados (la falta de rendición de cuentas), se utiliza la autoridad del Estado para proteger actividades delic- tivas y hay estrechos vínculos entre las agrupaciones delictivas y las élites políticas, o la falta de autoridad frente al crimen organizado o el exceso de autoridad sobre la población indefensa...». Y concluye: «La democratización no es todavía a escala universal un criterio de estatalidad o condición de reconocimiento pues la democracia es un proceso que requiere convicción, tiempo y esfuerzo en su seno. Sin embargo, se puede constatar que es un valor meta para esos pueblos y que sirve al valor universal más preciado, la paz interna e internacional. Muchos Estados están lejos de haber alcanzado ese ideal pero la tendencia es ya un progreso y siempre una esperanza frente a la frustrante decepción de aquellas democracias en las que, tras hacer transiciones difíciles, la corrupción y la partitocracia se aliaron en la deconstrucción del Estado de Derecho poniendo en peligro, junto al tribalismo nacionalista, la existencia misma del Estado».

8. Por último, se ocupa del principio del Estado de Derecho como otro valor esencial, pues «un Estado que se someta a las normas internas democráticas bajo control jurisdiccional y a las normas internacionales bajo los controles de las organizaciones internacionales y tribunales internacionales es un factor de paz considerable», aunque en la actualidad la exigencia de este principio como obligación internacional de cada Estado es un objetivo y una tendencia pero está todavía lejos de la obligación constitucional interna propia de los Estados democráticos. De otro lado, la práctica pone de relieve la presencia de factores de erosión del Estado de Derecho en el plano internacional e incluso en el interno, como sucede con algunas de las medidas tomadas en la lucha contra el terrorismo internacional; en este punto, la Prof. a Mangas destaca cómo el Tribunal de Justicia de la Unión Europea "puso algo de sensatez», pues «el Consejo de Seguridad se olvidó de algo bien simple: que la lucha contra el terrorismo requiere, más que en ninguna 
otra circunstancia, el control judicial de los actos del poder. Todos, en especial los poderosos, bajo el imperio del Derecho».

9. En la Real Academia de Ciencias Morales y Políticas, el discurso de ingreso de la Prof. ${ }^{a}$ Mangas Martín fue toda una defensa de un determinado ethos en la comunidad internacional; toda una lección moral. Con toda razón, en su discurso de contestación, Santiago Muñoz Machado destacó su carrera honesta y brillante, servicial y productiva. Es de justicia añadir su compromiso ético.

Paz Andrés SÁEnz de Santa María Universidad de Oviedo

http://dx.doi.org/10.17103/redi.67.1.2015.5.04

Pérez González, M. (dir.) y Conde Pérez, E. (coord.), Lucha contra el terrorismo, Derecho internacional humanitario y Derecho penal internacional, Valencia, Tirant lo Blanch, 2012, 363 pp.

1. No resulta fácil hacer la recensión de un libro que incluye diez aportaciones de autores distintos, pero el prestigio y la autoridad académica de su director, la calidad y el rigor científico de los trabajos y el interés cada vez mayor de los temas tratados animan al autor de estas líneas a una sucinta presentación de conjunto y a una breve valoración de la obra.

2. En cuanto a los autores hay que decir que unos son acreditados académicos en la Universidad española, a saber, Eduardo Vilariño, Montserrat Abad, Elena Conde, Carmela Pérez y Ruth Abril, amigos e integrantes todos ellos del equipo científico del profesor que dirige la publicación. Otros colaboradores, no menos prestigiosos y valiosos en las disciplinas centrales del libro - Derecho internacional humanitario y Derecho penal internacional-, son Fernando Pignatelli, José Luis Rodríguez Villasante, Pasquale de Sena, Marco Pedrazzi y Milena Costas.

3. El tema central del libro es el terrorismo, realidad pavorosa, de incierta definición, pero en todo caso susceptible de análisis desde varias disciplinas científicas, a saber, la ética, el Derecho, la ciencia política y otras. De todos modos, escrito el libro por enseñantes y profesionales del Derecho, prevalece en sus páginas el enfoque jurídico, aunque ello sin perjuicio del necesario enfoque interdisciplinar. Y los sectores específicos de la normativa internacional que se toman en consideración son los relativos al uso de la fuerza -ius ad bellum-, al Derecho internacional humanitario -ius in bello-, al Derecho internacional de los derechos humanos y al Derecho internacional penal, ramas todas ellas presentes e interconectadas en el tema del terrorismo y en muchos otros temas de la arena internacional.

4. Es bien sabido por lo demás que, lamentablemente, las respuestas de los Estados a los actos terroristas no siempre se han conformado a las exigencias del Derecho internacional de los derechos humanos. Los ejemplos de graves violaciones de esa normativa están en la mente de todos y no parece necesario mencionarlos. Pero hay que insistir en la idea de que semejante actitud constituye no sólo una palmaria y reprobable vulneración de los derechos fundamentales, sino también un error de gran calibre, porque el terror no se combate con más terror, el terrorismo de Estado, y aunque las manifestaciones de éste sean de menor intensidad y gravedad. Terrorismo este último que no disuade a los terroristas de la comisión de nuevos atentados sino que, por el contrario, tomado como provocación, les anima a la perpetración de ulteriores crímenes.

5. Es de encomiar, en fin, la estrategia que se advierte en las diversas contribuciones al libro sobre la manera de combatir el terrorismo; estrategia inte- 
grada que se compondría de los siguientes elementos: prevención, protección, respuesta judicial y penal - nacional o internacional- y cooperación internacional institucionalizada. Y todo ello, es importante señalarlo, en el marco del respeto al Derecho internacional de los dere- chos humanos y al Derecho internacional humanitario. Excelente manera, en mi opinión, de ver y entender las cosas.

José Antonio PASTOR RIDRUEJO Universidad Complutense http://dx.doi.org/10.17103/redi.67.1.2015.5.05

\section{B) LIBROS RECIBIDOS}

Bou Franch, V. y Castillo Daudí, M., Derecho internacional de los derechos humanos y Derecho internacional humanitario, Valencia, Tirant lo Blanch, 2014, 623 pp.

Bou Franch, V. (dir.), Introducción al Derecho de la Unión Europea, Cizur Menor, Thomson Reuters, Civitas, 2014, 477 pp.

Gutiérrez Espada, C. y Cervell Hortal, M. J., Nacimiento, auge y decadencia de la responsabilidad de proteger, Granada, Comares, 2014, 201 pp.

Orihuela Calatayud, E., Las víctimas y la Corte Penal Internacional, Cizur Menor, Thomson Reuters, Aranzadi, 2014, 275 pp.

Pastor Ridruejo, J. A. y Acosta Alvarado, P. A., Los sistemas internacionales de protección de derechos humanos, Bogotá, Universidad Externado de Colombia, 2014, 125 pp. 